\title{
STATISTICS AND QUANTUM GROUP SYMMETRIES
}

\author{
GAETANO FIORE \\ Sektion Physik, Universität München, LS Prof. Wess \\ Theresienstr. 37, D-80333 München, Germany \\ E-mail: Gaetano.Fiore@physik.uni-muenchen.de,gfiore@na.infn.it \\ PETER SCHUPP \\ Sektion Physik, Universität München, LS Prof. Wess \\ Theresienstr. 37, D-80333 München, Germany \\ E-mail: Peter.Schupp@physik.uni-muenchen.de
}

\begin{abstract}
Using "twisted" realizations of the symmetric groups, we show that Bose and Fermi statistics are compatible with transformations generated by compact quantum groups of Drinfel'd type.

1. Introduction. In recent times quantum groups have been often suggested as candidates for generalized symmetry transformations in Quantum Field Theory. One way to reach QFT is to deform canonical commutation relations of fields in such a way that they become quantum group covariant [1]. Another way is first to find a consistent procedure to implement quantum group transformations in Quantum Mechanics with a finite number of identical particles and then to pass to QFT through second quantization; in this approach the "particle interpretation" of QFT is the starting point. In the present work we adopt this second approach and give a consistent first quantized construction $[2]$.

Are the notions of identical particles and quantum (group) symmetry compatible? In other words, can the Hilbert space of states of $n$ bosons/fermions carry both a representation of the symmetric group $S_{n}$ and of $H$ ? - One might think that this is impossible.

Let $H$ be some $*$ Hopf algebra and $\rho: H \rightarrow \mathcal{A}$ a realization of $H$ in a $*$-algebra $\mathcal{A}$ of operators that act on a one-particle Hilbert space $\mathcal{H}$. We say: "the system transforms under the action of $H$ ". If $H$ is not co-commutative, then the action of $H$ on $\mathcal{H} \otimes \mathcal{H}$, defined
\end{abstract}

1991 Mathematics Subject Classification: Primary 81R50; Secondary 17B37.

The first author supported by a postdoc fellowship of the A. v. Humboldt Foundation.

The paper is in final form and no version of it will be published elsewhere. 
by $\rho^{(2)}=(\rho \otimes \rho) \circ \Delta$, where $\Delta$ is the coproduct, does not preserve but rather mixes the symmetric and antisymmetric subspaces $(\mathcal{H} \otimes \mathcal{H})_{ \pm}$defined by $P_{12}(\mathcal{H} \otimes \mathcal{H})_{ \pm}= \pm(\mathcal{H} \otimes \mathcal{H})_{ \pm}$ respectively ( $P_{12}$ denotes the permutation operator). Fermions and bosons in the ordinary sense are impossible.

This is true even if $H$ is just a slight deformation of a co-commutative Hopf algebra, because a drastic and unacceptable discontinuity of the number of allowed states of the multi-particle system would appear in the limit of vanishing deformation parameter.

We want to show that there exists a way out, at least if $H$ is a compact section of a quantum group of Drinfel'd type, i.e. a quantization of a Poisson-Lie group.

The apparent incompatibility arises from two facts: 1) in the (ordinary) formulation of Quantum Mechanics one associates to each separate tensor factor one of the particles; 2) the coproduct does not "treat" different tensor factors symmetrically. In the following we want to show that point 1) is only a possibility, not a necessity.

2. Twisted symmetrization postulates. The "permutation group" $S_{n}$ enters the definitions of the Hilbert space and the algebra of operators of a system of bosons/fermions. The first step is to show that the elements of $S_{n}$ can be realized in a way other than by ordinary permutators.

The standard symmetry postulates characterizing a system of $\mathbf{2}$ bosons or fermions are:

$$
\begin{aligned}
& P_{12}|u\rangle_{ \pm}= \pm|u\rangle_{ \pm} \quad \text { for } \quad|u\rangle_{ \pm} \in(\mathcal{H} \otimes \mathcal{H})_{ \pm} \\
& a:(\mathcal{H} \otimes \mathcal{H})_{ \pm} \rightarrow(\mathcal{H} \otimes \mathcal{H})_{ \pm} \quad \text { for } \quad a \in(\mathcal{A} \otimes \mathcal{A})_{+} \\
& *_{2}:(\mathcal{A} \otimes \mathcal{A})_{+} \rightarrow(\mathcal{A} \otimes \mathcal{A})_{+},
\end{aligned}
$$

where $*_{2} \equiv * \otimes *$ and $(\mathcal{A} \otimes \mathcal{A})_{+}:=\left\{a \in \mathcal{A} \otimes \mathcal{A}:\left[P_{12}, a\right]=0\right\}$. Equation (1) defines bosonic $(+)$ and fermionic $(-)$ states. Equation $(2)$ follows from $\left[P_{12},(\mathcal{A} \otimes \mathcal{A})_{+}\right]=0$ and shows that symmetrized operators map boson (fermion) states into boson (fermion) states.

For a unitary (and in general not symmetric) operator $F_{12} \in \mathcal{A} \otimes \mathcal{A}, F_{12}^{* 2}=F_{12}^{-1}$ we define

$$
\begin{aligned}
(\mathcal{H} \otimes \mathcal{H})_{ \pm}^{F_{12}} & :=F_{12}(\mathcal{H} \otimes \mathcal{H})_{ \pm} \\
P_{12}^{F_{12}} & :=F_{12} P_{12} F_{12}^{-1} \\
(\mathcal{A} \otimes \mathcal{A})_{+}^{F_{12}} & :=F_{12}(\mathcal{A} \otimes \mathcal{A})_{+} F_{12}^{-1} \\
& =\left\{a \in \mathcal{A} \otimes \mathcal{A}:\left[P_{12}^{F_{12}}, a\right]=0\right\}
\end{aligned}
$$

with $(\mathcal{A} \otimes \mathcal{A})_{+}$as defined above. Note that $\left(P_{12}^{F_{12}}\right)^{2}=\mathrm{id}=\left(P_{12}\right)^{2}$. We find in complete analogy to equations $(1)-(3)$

$$
\begin{aligned}
& P_{12}^{F_{12}}|u\rangle_{ \pm}= \pm|u\rangle_{ \pm} \quad \text { for }|u\rangle_{ \pm} \in(\mathcal{H} \otimes \mathcal{H})_{ \pm}^{F_{12}} \\
& a:(\mathcal{H} \otimes \mathcal{H})_{ \pm}^{F_{12}} \rightarrow(\mathcal{H} \otimes \mathcal{H})_{ \pm}^{F_{12}} \text { for } a \in(\mathcal{A} \otimes \mathcal{A})_{+}^{F_{12}} \\
& *_{2}:(\mathcal{A} \otimes \mathcal{A})_{+}^{F_{12}} \rightarrow(\mathcal{A} \otimes \mathcal{A})_{+}^{F_{12}}
\end{aligned}
$$

and $a^{F_{12}}:=F_{12} a F_{12}^{-1}$ is hermitean iff $a$ is. Eq.'s $(7)-(9)$ are formally identical to eq.'s (1) - (3) and provide an alternative quantum mechanical description of a system of two 
bosons/fermions. The unitarity of $F_{12}$ guarantees that from the kinematical viewpoint the new "twisted" description is physically equivalent to the standard one.

The generalization to $\mathbf{n}$ bosons or fermions is straightforward: Let $F_{12 \ldots n} \in \mathcal{A}^{\otimes n}$ be unitary, i.e. $\left(F_{12 \ldots n}\right)^{*_{n}}=\left(F_{12 \ldots n}\right)^{-1}$, where $*_{n}:=*^{\otimes n}$, and define

$$
\begin{aligned}
(\mathcal{H} \otimes \ldots \otimes \mathcal{H})_{ \pm}^{F_{12 \ldots n}}: & =F_{12 \ldots n}(\mathcal{H} \otimes \ldots \otimes \mathcal{H})_{ \pm} \\
P_{12}^{F_{12 \ldots n}} & :=F_{12 \ldots n} P_{12}\left(F_{12 \ldots n}\right)^{-1} \\
\vdots & \\
P_{n-1, n}^{F_{12 \ldots n}}: & =F_{12 \ldots n} P_{n-1, n}\left(F_{12 \ldots n}\right)^{-1} \\
(\mathcal{A} \otimes \ldots \otimes \mathcal{A})_{+}^{F_{12 \ldots n}} & :=F_{12 \ldots n}(\mathcal{A} \otimes \ldots \otimes \mathcal{A})_{+}\left(F_{12 \ldots n}\right)^{-1}
\end{aligned}
$$

with $(\mathcal{A} \otimes \ldots \otimes \mathcal{A})_{+}:=\left\{a \in \mathcal{A} \otimes \ldots \otimes \mathcal{A}:\left[P_{i, i+1}, a\right]=0, i=1, \ldots n-1\right\}$, and $P_{i, i+1}$ the permutator of the $i^{t h},(i+1)^{t h}$ tensor factors. Then

$$
\begin{aligned}
& P_{i, i+1}^{F_{12 \ldots n}}|u\rangle_{ \pm}= \pm|u\rangle_{ \pm} \quad \text { for } \quad|u\rangle_{ \pm} \in(\mathcal{H} \otimes \ldots \otimes \mathcal{H})_{ \pm}^{F_{12 \ldots n}} \\
& a:(\mathcal{H} \otimes \ldots \otimes \mathcal{H})_{ \pm}^{F_{12 \ldots n}} \rightarrow(\mathcal{H} \otimes \ldots \otimes \mathcal{H})_{ \pm}^{F_{12 \ldots n}} \\
& \text { for } a \in(\mathcal{A} \otimes \ldots \otimes \mathcal{A})_{+}^{F_{12 \ldots n}} \\
& *_{n}:(\mathcal{A} \otimes \ldots \otimes \mathcal{A})_{+}^{F_{12 \ldots n}} \rightarrow(\mathcal{A} \otimes \ldots \otimes \mathcal{A})_{+}^{F_{12 \ldots n}} .
\end{aligned}
$$

Equation (14) follows from $\left[P_{i, i+1}^{F_{12 \ldots n}},(\mathcal{A} \otimes \ldots \otimes \mathcal{A})_{+}^{F_{12 \ldots n}}\right]=0$.

The operators $P_{i, j}^{F_{12 \ldots n}}$ satisfy the same algebraic relations as the $P_{i, j}$. They therefore provide a different realization of the group $S_{n}$. Eq.'s $(13)-(15)$ give an alternative quantum mechanical description of a system of $n$ bosons/fermions; note that in the latter equations the twist $F_{12 \ldots n}$ does not appear explicitly any more.

3. Identical versus distinct particles. It is every day's experience that in many situations identical particles can be equivalently treated as distinct. If this were not the case, one could not describe some particles (in our "laboratory", say) without describing (with a single huge wave-function) all the other particles of the same kind in the universe.

There exists a precise correspondence between these two descriptions in the standard quantum mechanical formalism; in the twisted approach the twist $F$ directly enters the rule governing this correspondence.

Consider as gedanken experiment the scattering of two identical particles. One can distinguish three stages: In the initial stage the two particles are far apart, i.e. are prepared in two separate orthonormal one-particle states $\left|\psi_{1}\right\rangle,\left|\psi_{2}\right\rangle$. In the intermediate stage, the particles approach each other and scatter. In the final stage, long after the collision, the particles are again far apart and are detected by one-particle detectors. Since the preparation and measurement are essentially one-particle processes, we need to:

(a) Translate the initial data (two one-particle states $\left|\psi_{1}\right\rangle,\left|\psi_{2}\right\rangle$ ) into a properly (anti-)symmetrized two-particle state $|\psi\rangle$; in fact, the collision is correctly described only if we apply a symmetric evolution operator to such a state.

(b) Translate the final (anti-)symmetrized two-particle state $\left|\psi^{\prime}\right\rangle$ into one-particle data, i.e. a superposition $\left|\psi_{d}^{\prime}\right\rangle$ of (correlated) orthonormal one-particle states. 
In the twisted case these translations are done according to the following rule: $|\psi\rangle$ is obtained by

$$
|\psi\rangle:=\frac{F_{12}}{\sqrt{2}}\left(\left|\psi_{1}\right\rangle \otimes\left|\psi_{2}\right\rangle \pm\left|\psi_{2}\right\rangle \otimes\left|\psi_{1}\right\rangle\right) \in(\mathcal{H} \otimes \mathcal{H})_{ \pm}^{F_{12}}
$$

and, with the final state of the form $\left|\psi^{\prime}\right\rangle=\sum_{i}, \frac{F_{12}}{\sqrt{2}}\left(\left|\psi_{1}^{i}\right\rangle \otimes\left|\psi_{2}^{i}\right\rangle \pm\left|\psi_{2}^{i}\right\rangle \otimes\left|\psi_{1}^{i}\right\rangle\right)$, where $\left\langle\psi_{1}^{i} \mid \psi_{2}^{i}\right\rangle=0,\left|\psi_{d}^{\prime}\right\rangle$ is obtained as

$$
\left|\psi_{d}^{\prime}\right\rangle=\sum_{i}\left|\psi_{1}^{i}\right\rangle \otimes\left|\psi_{2}^{i}\right\rangle
$$

4. Quantum symmetries. While their introduction was shown to be consistent, there was so far no need for the $F_{12 \ldots n}$ 's. Now we take the issue of quantum group symmetries into consideration. We require that multi-particle systems transform under Hopf algebra actions in a way that is consistent with twisted (anti-)symmetric states and operators. Such a twist $F_{12 \ldots n}$ will be strictly related to the coproduct $\Delta$. A trivial $F_{12 \ldots n}$ is only possible for a cocommutative $\Delta$.

4.1. Transformation of states and operators. The transformation $\triangleright$ of $\mathcal{H}$ under $H$ shall be given by a $*$-algebra homomorphism $\rho: H \rightarrow \mathcal{A}$ with $\rho(x) \rho(y)=\rho(x y), \rho\left(x^{*}\right)=\rho^{\dagger}(x)$, and $\rho(1)=\mathbf{1}$ :

$$
|\psi\rangle \rightarrow \rho(x)|\psi\rangle=: x \triangleright|\psi\rangle, \quad|\psi\rangle \in \mathcal{H}, \quad x \in H .
$$

The corresponding transformation of operators under the action of $H$ is

$$
\mathcal{O} \rightarrow \rho\left(x_{(1)}\right) \mathcal{O} \rho\left(S x_{(2)}\right)=: x \triangleright \mathcal{O}, \quad x_{(1)} \otimes x_{(2)} \equiv \Delta(x) \in H \otimes H, \quad \mathcal{O} \in \mathcal{A} .
$$

The transformation of multi particle systems, i.e. of $\left|\psi^{(n)}\right\rangle \in \mathcal{H}^{\otimes n}$ and $\mathcal{O}^{(n)} \in \mathcal{A}^{\otimes n}$ is obtained simply by replacing $\rho$ in expressions (18) and (19) by

$$
\rho^{(n)}=\rho^{\otimes n} \circ \Delta^{(n-1)} .
$$

The reader may wonder what happened to the ordinary commutator (i.e. the adjoint action in the undeformed setting): The commutator is in general distinct from the quantum adjoint action (19), but it still plays a role here: An operator $\mathcal{O}$ is symmetric (invariant) under the transformations generated by $h \subset H$ if $x \triangleright \mathcal{O}=\epsilon(x) \mathcal{O}$ for all $x \in h$; it may be simultaneously diagonalizable with elements $x \in h$ if $[\rho(x), \mathcal{O}]=0$. Symmetry and vanishing commutator coincide if $\Delta(h) \subset h \otimes H$.

The transformation of $\mathcal{H}$ and $\mathcal{A}$ under the Hopf algebra $H$ should be consistent with the twisted symmetrization postulates of section 2: We hence require that

$$
\begin{aligned}
& \triangleright: H \times\left(\mathcal{H}^{\otimes n}\right)_{ \pm}^{F_{12 \ldots n}} \rightarrow\left(\mathcal{H}^{\otimes n}\right)_{ \pm}^{F_{12 \ldots n}}, \\
& \triangleright: H \times\left(\mathcal{A}^{\otimes n}\right)_{+}^{F_{12 \ldots n}} \rightarrow\left(\mathcal{A}^{\otimes n}\right)_{+}^{F_{12 \ldots n}}
\end{aligned}
$$

for a suitably chosen $F_{12 \ldots n}$. The conditions to be satisfied are

$$
\left[\rho^{(n)}(x), P_{i, i+1}^{F_{12 \ldots n}}\right]=0, \quad \forall x \in H, \quad i=1, \ldots, n-1,
$$

because then the action of $H$ will commute with the twisted permutations $P_{i, i+1}^{F_{12 \ldots n}}$ :

$$
P^{F}\left(x \triangleright\left|\psi^{(n)}\right\rangle\right)=P^{F} \rho^{(n)}(x)\left|\psi^{(n)}\right\rangle=\rho^{(n)}(x)\left(P^{F}\left|\psi^{(n)}\right\rangle\right)=x \triangleright\left(P^{F}\left|\psi^{(n)}\right\rangle\right)
$$


and

$$
\begin{aligned}
{\left[P^{F}, x \triangleright \mathcal{O}^{(n)}\right] } & =\left[P^{F}, \rho^{(n)}\left(x_{(1)}\right) \mathcal{O}^{(n)} \rho^{(n)}\left(S x_{(2)}\right)\right]=\rho^{(n)}\left(x_{(1)}\right)\left[P^{F}, \mathcal{O}^{(n)}\right] \rho^{(n)}\left(S x_{(2)}\right) \\
& =x \triangleright\left[P^{F}, \mathcal{O}^{(n)}\right]
\end{aligned}
$$

(Here we have suppressed the indices on $P^{F}$.)

Conditions (23) are equivalent to requiring

$$
\rho^{(n)}(H) \subset\left(\mathcal{A}^{\otimes n}\right)_{+}^{F_{12 \ldots n}}
$$

for some $F_{12 \ldots n}$. This is certainly satisfied if

$$
\rho^{(n)}(H)=F_{12 . . n} \rho_{c}^{(n)}(H) F_{12 . . n}^{-1},
$$

where $\rho_{c}^{(n)}:=\rho^{\otimes n} \circ \Delta_{c}^{(n-1)}$ and $\Delta_{c}$ is a cocommutative coproduct. The following theorem will be our guidance in finding the right $F$ 's when $H=U_{q}(g)$.

\subsection{Drinfeld Proposition 3.16 in [3]}

1. There exists an algebra isomorphism $\phi: U_{q} \mathbf{g} \tilde{\leftrightarrow}(U \mathbf{g})([[h]])$, where $h=\ln q$ is the deformation parameter.

2. If we identify the isomorphic elements of $U_{q} \mathbf{g}$ and $(U \mathbf{g})([[h]])$ then there exists an $\mathcal{F} \in U_{q} \mathbf{g} \otimes U_{q} \mathbf{g}$ such that:

$$
\Delta(a)=\mathcal{F} \Delta_{c}(a) \mathcal{F}^{-1}, \quad \forall a \in U_{q} \mathbf{g} \cong(U \mathbf{g})([[h]])
$$

where $\Delta$ is the coproduct of $U_{q} \mathbf{g}$ and $\Delta_{c}$ is the (co-commutative) coproduct of $U(\mathbf{g})$.

3. $(U \mathbf{g})([[h]])$ is a quasi-triangular quasi-Hopf algebra $(Q T Q H A)$ with universal $\mathcal{R}_{\Phi}=$ $q^{t / 2}$ and a quasi-coassociative structure given by an element $\Phi \in\left((U \mathbf{g})^{\otimes 3}([[h]])\right)$ that is expressible in terms of $\mathcal{F} .(U \mathbf{g})([[h]])$ as QTQHA can be transformed via the twist by $\mathcal{F}$ into the quasi-triangular Hopf algebra $U_{q} \mathbf{g}$; in particular, the universal $\mathcal{R}$ of $U_{q} \mathbf{g}$ is given by $\mathcal{R}=\mathcal{F}_{21} \mathcal{R}_{\Phi} \mathcal{F}^{-1}$.

If $H$ is the quantization of a Poisson-Lie group associated with a solution of the classical Yang-Baxter equation (CYBE) $\left(^{1}\right)$ then another theorem [4] states the existence of an $\mathcal{F}$ with similar properties as in the previous theorem - except that now it is enough to twist $(U \mathbf{g})([[h]])$ equipped with the ordinary coassociative structure in order to obtain $H$.

As shown in [5] one can always choose $\mathcal{F}$ to be unitary, as long as $H$ is a compact section of $U_{q} \mathbf{g}$ (i.e.when $q \in \mathbf{R}$ ). The theorems suggest that one can use the unitary twisting operator $\mathcal{F}$ to build $F_{12}$ for a 2-particle sytem.

Examples. 1. If $\mathcal{A}=\rho\left(U_{q} \mathbf{g}\right)$, then we choose

$$
F=\rho^{\otimes 2}(\mathcal{F}) \text {. }
$$

Ex.: $H=U_{q}(s u(2))$, deformed quantum rotator.

2. If $\mathcal{A}=$ Univ. covering Poincaré $\otimes \rho\left(U_{q} \mathbf{g}\right)$, were $U_{q} \mathbf{g}$ plays the role of an internal symmetry, then we can set

$$
F_{12}=\mathrm{id}_{\text {Poincaré }}^{(2)} \otimes \rho^{\otimes 2}(\mathcal{F}) .
$$

$\left.{ }^{1}\right)$ In this case $H$ is is triangular, i.e. $\mathcal{R}_{21} \mathcal{R}_{12}=\mathbf{1}$. 
3. If $\mathcal{A}$ is the q-deformed Poincaré algebra of ref. [6,7], and $H$ is the corresponding q-deformed Lorentz Hopf algebra, realized through $\rho$ in $\mathcal{A}$, then we can again define

$$
F_{12}=\rho^{\otimes 2}(\mathcal{F})
$$

where $\mathcal{F}$ belongs to $H \otimes H$. The same applies for other inhomogeneous algebras, like the q-Euclidean ones, constructed from the braided semi-direct product [7] of a quantum space and of the corresponding homogeneous quantum group. For both of these examples the one-particle representation theory is known $[8,9]$.

For $n$-particle systems one can find $F_{12 \ldots n}$ by replacing in the previous equations $\mathcal{F}$ by one particular element $\mathcal{F}_{12 \ldots n}$ of $H^{\otimes n}$ satisfying the condition

$$
\Delta(x)=\mathcal{F}_{12 \ldots n} \Delta_{c}(x)\left(\mathcal{F}_{12 \ldots n}\right)^{-1} .
$$

To obtain one such $\mathcal{F}_{12 \ldots n}$ it is enough to act on eq. $(28)(n-2)$ times with the coproduct in some arbitrary order. When $n=3$, for instance, one can use

$$
\text { either } \quad \mathcal{F}_{123}^{\prime}:=[(\Delta \otimes i d)(\mathcal{F})] \mathcal{F}_{12} \quad \text { or } \quad \mathcal{F}_{123}^{\prime \prime}:=[(i d \otimes \Delta)(\mathcal{F})] \mathcal{F}_{23} .
$$

They coincide if $H$ is the quantization of a solution of the CYBE [Drinfeld]. In the case of $U_{q} \mathbf{g}$, they do not coincide, but nevertheless $\Phi:=\mathcal{F}_{123}^{\prime \prime}\left(\mathcal{F}_{123}^{\prime}\right)^{-1} \neq \mathbf{1} \otimes \mathbf{1} \otimes \mathbf{1}$ commutes with $\Delta^{(2)}(H)$, implying that both satisfy eq. (29). One can find a continuous family of $\mathcal{F}_{123}$ interpolating between $\mathcal{F}_{123}^{\prime}$ and $\mathcal{F}_{123}^{\prime \prime} .(\mathcal{A} \otimes \mathcal{A} \otimes \mathcal{A})_{+}^{F_{123}}$ will depend on the specific choice of $\mathcal{F}_{123}$; at this stage, no a priori preferred choice for $\mathcal{F}_{123}$ is known.

Not e. From Eq. (28) follows $(\tau \circ \Delta)(a)=\mathcal{M} \Delta(a) \mathcal{M}^{-1}$ with $\mathcal{M}:=\mathcal{F}_{21} \mathcal{F}^{-1}$. This is not the usual relation $(\tau \circ \Delta)(a)=\mathcal{R} \Delta(a) \mathcal{R}^{-1}$ of a quasi-triangular Hopf algebra; the latter is rather obtained by rewriting equation (28) in the form $\Delta(a)=\mathcal{F} q^{t / 2} \Delta_{c}(a) q^{-t / 2} \mathcal{F}^{-1}$ where $t=\Delta_{c}\left(C_{c}\right)-1 \otimes C_{c}-C_{c} \otimes 1$ is the invariant tensor $\quad\left(\left[t, \Delta_{c}(a)\right]=0 \forall a \in U \mathbf{g}\right)$ corresponding to the Killing metric, and $C_{c}$ is the quadratic casimir of $U \mathbf{g} . \mathcal{M}$, unlike $\mathcal{R}$, has not nice properties under the coproducts $\Delta \otimes i d, i d \otimes \Delta$.

The reader might wonder whether we could use equation $\left[P_{12} R,(\mathcal{A} \otimes \mathcal{A})_{+}^{\prime}\right]=0$ (where $\left.R=\rho^{\otimes 2}(\mathcal{R})\right)$, instead of eq. (6), to single out a modified symmetric algebra $(\mathcal{A} \otimes \mathcal{A})_{+}^{\prime} \subset$ $\mathcal{A} \otimes \mathcal{A}$; in fact, the former is also an equation fulfilled by $\rho^{\otimes 2}(\Delta(H))$ and reduces to the classical eq. $\left[P_{12},(\mathcal{A} \otimes \mathcal{A})_{+}^{\prime}\right]=0$ in the limit $q \rightarrow 1$. However $\left[P_{12} R,(\mathcal{A} \otimes \mathcal{A})_{+}^{\prime}\right]=0$ is fulfilled only by the sub-algebra $\rho^{\otimes 2}(\Delta(H)) \subset(\mathcal{A} \otimes \mathcal{A})$, essentially because $q^{t / 2}$ does not commute with all symmetric operators, but only with the ones corresponding to coproducts. Therefore, $(\mathcal{A} \otimes \mathcal{A})_{+}^{\prime}$ is not big enough to be in one-to-one correspondence with the classical $(\mathcal{A} \otimes \mathcal{A})_{+}$, i.e. is not suitable for our purposes.

Unfortunately, explicit universal $\mathcal{F}$ 's for $U_{q} \mathbf{g}$ are not given in the literature; a $\mathcal{F}$ for a family of deformations (which include quantizations of solutions of both of a CYBE and of a MCBYE) of the Heisenberg group in one dimension was given in Ref. [10].

However, for most practical purposes one has to deal with representations $F$ of $\mathcal{F}$. In Ref. [11] a straightforward method for finding the matrix representations of $\mathcal{F}$ from those of $\mathcal{R}$ was proposed and explicit formulas were given for the $A, B, C, D$-series in the fundamental representation. In [12] matrices twisting the classical coproduct into the q-deformed one, and therefore related to Drinfeld's twist, were already found. 
Moreover, in the intrinsic formulation of the twisted (anti-)symmetrization postulates [eqs. (13) - (15)] one only needs the twisted permutators $P_{12 \ldots n}^{F_{12 \ldots n}}$ (not the $F_{12 \ldots n}$ themselves); explicit universal expressions for the latter may be found much more easily, as we will show for $P_{12}^{\mathcal{F}_{12}}$ in the case $H=U_{q}(s u(2))$.

5. Explicit example: $H=U_{q}(s u(2))$. Let the one-particle system be a q-deformed rotator: $\mathcal{A} \equiv \rho(H):=\rho\left[U_{q}(s u(2))\right]$, with $q \in \mathbf{R}^{+}$. Consider an irreducible $*$-representation of $H$, namely $\mathcal{H} \equiv V_{j}$, where $V_{j}$ denotes the highest weight representation of $U_{q}(s u(2))$ with highest weight $j=0, \frac{1}{2}, 1, \ldots$.

5.1. The case of two particles. What are $(\mathcal{H} \otimes \mathcal{H})_{ \pm}^{F_{12}}$ and $(\mathcal{A} \otimes \mathcal{A})_{+}^{F_{12}}$ ?

Let us identify $U_{q}(s u(2))$ and $U(s u(2))$ as algebras through the isomorphism $\phi$ of the first point of Drinfeld's theorem; as a consequence, $V_{j}$ carries a representation $\rho$ of both algebras. Similarly, $V_{j} \otimes V_{j}$ carries (irreducible) representations of both $U_{q}(s u(2)) \otimes$ $U_{q}(s u(2))$ and $U(s u(2)) \otimes U(s u(2))$, as well as (reducible) representations $\rho_{c}^{(2)}(X)$ and $\rho^{(2)}$ of $U_{q}(s u(2))$ and $U(s u(2))$ respectively (by definition $\rho^{(2)}(X):=\rho^{\otimes 2}[\Delta(X)]$ and $\left.\rho_{c}^{(2)}(X):=\rho^{\otimes 2}\left[\Delta_{c}(X)\right]\right)$. Let $\mathcal{V}_{J}$ and $\mathcal{V}_{J}^{q}(0 \leq J \leq 2 j)$ respectively denote the carrier spaces of the irreducible components of the latter; from the second point of Drinfeld's theorem it follows that

$$
F_{12} \mathcal{V}_{J}=\mathcal{V}_{J}^{q}
$$

Recall now that the $\mathcal{V}_{J}$ 's have well-defined symmetry w.r.t the permutation, namely

$$
\mathcal{V}_{2 j-k} \text { is }\left\{\begin{array} { l } 
{ \text { symmetric } } \\
{ \text { antisymmetric } }
\end{array} \text { if } k \text { is } \left\{\begin{array}{l}
\text { even } \\
\text { odd }
\end{array} .\right.\right.
$$

Therefore

$$
\begin{aligned}
& \left(V_{j} \otimes V_{j}\right)_{+}^{F_{12}}:=F_{12}\left(V_{j} \otimes V_{j}\right)_{+}=F_{12}\left(\bigoplus_{0 \leq l \leq j} \mathcal{V}_{2(j-l)}\right)=\bigoplus_{0 \leq l \leq j} \mathcal{V}_{2(j-l)}^{q} \\
& \left(V_{j} \otimes V_{j}\right)_{-}^{F_{12}}:=F_{12}\left(V_{j} \otimes V_{j}\right)_{-}=F_{12}\left(\bigoplus_{0 \leq l \leq j-\frac{1}{2}} \mathcal{V}_{2(j-l)-1}\right)=\bigoplus_{0 \leq l \leq j-\frac{1}{2}} \mathcal{V}_{2(j-l)-1}^{q} .
\end{aligned}
$$

We can express the content of this equation by saying that the subspaces $\mathcal{V}_{J}^{q} \subset V_{j} \otimes V_{j}$ have well-defined "twisted symmetry".

Let us stress the difference between $(H \otimes H)_{+}^{F_{12}}$ and its sub-algebra $\rho^{(2)}(H)$ :

$$
\begin{aligned}
& \rho^{(2)}(H) \ni a: \mathcal{V}_{J}^{q} \rightarrow \mathcal{V}_{J}^{q}, \\
&(H \otimes H)_{+}^{F_{12}} \ni b:\left(V_{j} \otimes V_{j}\right)_{ \pm}^{F_{12}} \rightarrow\left(V_{j} \otimes V_{j}\right)_{ \pm}^{F_{12}} .
\end{aligned}
$$

The elements of $[\rho(H) \otimes \rho(H)]_{+} \backslash \rho^{(2)}(H)$ will in general map $\mathcal{V}_{J}^{q}$ out of itself, into some $\mathcal{V}_{J^{\prime}}^{q}$ 's with $J^{\prime} \neq J$.

5.2. Universal expression for the twisted permutation operator of $U_{q}(s u(2))$. We will say that an object $P_{12}^{\mathcal{F}_{12}}$ is the "universal twisted permutator" of $U_{q} \mathbf{g}$ if $P_{12}^{F_{12}}=\rho^{\otimes 2}\left(P_{12}^{\mathcal{F}_{12}}\right)$ for each representation $\rho$ of $U_{q} \mathbf{g}$.

In Ref. [2] we have shown that in the $\mathbf{g}=s u(2)$ case

$$
P_{12}^{\mathcal{F}_{12}}=f\left(\mathbf{1} \otimes C_{q}\right) f\left(C_{q} \otimes \mathbf{1}\right)\left[f\left(\Delta\left(C_{q}\right)\right)\right]^{-1} \mathcal{R}_{21} \circ \tau
$$


Here $\tau$ is the abstract permutator $(\tau a \otimes b=b \otimes a)$, and

$$
C_{q}=X^{-} X^{+}+\left(\frac{q^{\frac{h+1}{2}}-q^{\frac{-h-1}{2}}}{q-q^{-1}}\right)^{2}
$$

is the casimir of $U_{q}(s u(2))$ with eigenvalues $\left(\left[j+\frac{1}{2}\right]_{q}\right)^{2}$. In the limit $q \rightarrow 1: C_{q} \rightarrow C_{c}+\frac{1}{4}$, where $C_{c}$ is the usual casimir of $U(s u(2))$ with eigenvalues $j(j+1) . f(z)$ is defined by

$$
\log _{q}[f(z)]:=\left\{\frac{1}{\ln (q)} \sinh ^{-1}\left[\frac{\left(q-q^{-1}\right) \sqrt{z}}{2}\right]\right\}^{2}-\frac{1}{4}
$$

it is easy to verify that $f\left(C_{q}\right)$ has eigenvalues $q^{j(j+1)}$.

(For the proof of eq. (37), one uses the fact that the base space of each irreducible of representation contained in a tensor product is an eigenspace of $\mathcal{R}=\mathcal{F}_{21} q^{\frac{t}{2}} \mathcal{F}_{12}^{-1}$ with some eigenvalue $q^{\frac{1}{2}\left[J(J+1)-j_{1}\left(j_{1}+1\right)-j_{2}\left(j_{2}+1\right)\right]}$; here $t=\Delta_{c}\left(C_{c}\right)-C_{c} \otimes \mathbf{1}-\mathbf{1} \otimes C_{c}$, and $\left.j_{1}, j_{2}=0,1, \ldots, 0 \leq J \leq j_{1}+j_{2}\right)$.

5.3. The case of $n \geq 3$ particles. When $n \geq 3$, for any given space $V$ the decomposition of $\otimes^{n} V$ into irreducible representations of the permutation group contains components with partial or mixed symmetry, beside the completely symmetric and the completely antisymmetric ones.

As in the standard formulation, also in the twisted one the explicit knowledge of components with mixed or partial symmetry is required to build $\left(\mathcal{H}^{\otimes n}\right)_{ \pm}$if the Hilbert space $\mathcal{H}$ of one particle is the tensor product of different spaces corresponding to different degrees of freedom, $\mathcal{H}=V \otimes V^{\prime}$, as in examples 2. and 3. of section 4 .

6. Final remarks. 1. We have only discussed kinematics. To discuss dynamics we should introduce some Hamiltonian. Depending on the choice of Hamiltonian we find new physics or standard physics - although in a unusual picture; the latter situation would occur e.g. if the Hamiltonian is the twist-conjugate of a typical Hamiltonian describing a system with an ordinary group symmetry.

2. To generalize the above construction to non-compact real sections of quantum groups (of Drinfel'd type) one should consider also their infinite-dimensional representations and investigate whether the corresponding twist $\mathcal{F}$ can be realized as some unitary operator on them.

3. To introduce particle creation/annihilation operators $A_{i}^{\mathcal{F}_{12}}, A_{i}^{+\mathcal{F}_{12}}$ connecting twisted boson/fermion wave functions (second quantization) one can twist the ordinary creation/annihilation operators $A_{i}, A_{i}^{+}$through the unitary operator

$$
\mathcal{F}:=i d \oplus \operatorname{id} \oplus \mathcal{F}_{12} \oplus \mathcal{F}_{123} \oplus \ldots
$$

defined on the direct sum of all $m$-particle Hilbert spaces, $m=0,1,2, \ldots$. This will lead to canonical commutation relations for $A_{i}^{\mathcal{F}_{12}}, A_{i}^{+\mathcal{F}_{12}}$, but these operators will not transform covariantly under the quantum group action. In a separate publication we plan to show how in the twist formalism one can define quantum group covariant creation/annihilation operators; as a consequence, they will satisfy deformed commutation relations. In the $U_{q}(s u(N))$ case the result should coincide with the one previously found in Ref. [1]. 
Acknowledgments. We would like to thank W. Pusz and S. L. Woronowicz for pointing out reference [1].

\section{References}

[1] W. Pusz, S. L. Woronowicz, Twisted Second Quantization, Reports on Mathematical Physics 27 (1989), 231-257.

[2] G. Fiore, P. Schupp, Identical Particles and Quantum Symmetries, Preprint LMUTPW 95-10 (Munich University), hep-th 9508047, to appear in Nucl. Phys. B.

[3] V. G. Drinfeld, Quasi Hopf Algebras, Leningrad Math. J. 1 (1990), 1419.

[4] V. G. Drinfeld, Doklady AN SSSR 273 (1983) (in Russian), 531-535.

[5] B. Jurco, More on Quantum Groups from the Quantization Point of View, Commun. Math. Phys. 166 (1994), 63.

[6] O. Ogievetsky, W. B. Schmidke, J. Wess and B. Zumino, q-Deformed Poincaré Algebra, Commun. Math. Phys. 150 (1992) 495-518.

[7] S. Majid, Braided Momentum in the q-Poincaré Group, J. Math. Phys. 34 (1993), 2045.

[8] M. Pillin, W. B. Schmidke and J. Wess, q-Deformed Relativistic One-Particle States, Nucl. Phys. B403 (1993), 223.

[9] G. Fiore, The Euclidean Hopf algebra $U_{q}\left(e^{N}\right)$ and its fundamental Hilbert space representations, J. Math. Phys. 36 (1995), 4363-4405; The q-Euclidean algebra $U_{q}\left(e^{N}\right)$ and the corresponding q-Euclidean lattice, Int. J. Mod. Phys. A, in press.

[10] F. Bonechi, R. Giachetti, E. Sorace, M. Tarlini, Deformation Quantization of the Heisenberg Group, Commun. Math. Phys. 169 (1995), 627-633.

[11] R. Engeldinger, On the Drinfel'd-Kohno Equivalence of Groups and Quantum Groups, Preprint LMU-TPW 95-13.

[12] T. L. Curtright, G. I. Ghandour, C. K. Zachos, Quantum Algebra Deforming Maps, Clebsh-Gordan Coefficients, Coproducts, U and R Matrices, J. Math. Phys. 32 (1991), 676-688. 